\title{
Intensity coding of auditory stimuli: an fMRI study
}

\author{
L. JÄNCKE, ${ }^{\dagger}+$ N. J. SHAH, ${ }^{*}$ S. POSSE, ${ }^{*}$ M. GROSSE-RYUKEN* and \\ H.-W. MÜLLER-GÄRTNER* \\ * Research Center Jülich, Institute of Medicine, Jülich, Germany; † Institute of General Psychology, \\ Otto-von-Guericke-University-Magdeburg, Germany
}

(Received 10 September 1997; accepted 20 February 1998)

\begin{abstract}
The effect of stimulus intensity (sound pressure level, SPL) of auditory stimuli on the BOLD response in the auditory cortex was investigated in 14 young and healthy subjects, with no hearing abnormalities, using echo-planar, functional magnetic resonance imaging (fMRI) during a verbal and a non-verbal auditory discrimination task. The stimuli were presented block-wise at three different intensities: 95, 85 and $75 \mathrm{~dB}$ (SPL). All subjects showed fMRI signal increases in superior temporal gyrus (STG) covering primary and secondary auditory cortex. Most importantly, the spatial extent of the fMRI response in STG increased with increasing stimulus intensity. It is hypothesized that spreading of excitation is associated with the encoding of increasing stimulus intensity levels. In addition, we found bifrontal activation supposedly evoked by the auditory-articulary loop of working memory. The results presented here should assist in the design of optimal activation strategies for studying the auditory cortex with fMRI paradigms and may help in understanding intensity coding of auditory stimuli. (C) 1998 Elsevier Science Ltd. All rights reserved.
\end{abstract}

Key Words: cerebral blood flow; auditory cortex; functional imaging; acoustic perception; temporal lobe; magnetic resonance imaging; functional magnetic resonance imaging.

\section{Introduction}

Non-invasive methods such as functional magnetic resonance imaging (fMRI) and positron emission tomography (PET) are becoming more and more widespread for the study of sensory or cognitive functions. The BOLD (blood oxygen level dependent) contrast fMRI method measures regional changes of the nuclear magnetic resonance signal during brain activity. It is generally accepted that increased blood flow and oxygenation at the capillary venous level in activated tissue lead to decreased intravoxel dephasing, resulting in increased signal. In a series of studies it was shown that fMRI is also useful to study auditory perception processes despite the ambient masking noise from the scanner [3, 4, 6, 8]. However, data regarding the elementary stimulus variables (intensity, rate, duration) determining cerebral blood flow responses have been relatively few. For instance, rate of stimulus presentation has been shown to be a significant variable determining regional cerebral

$\$$ Author to whom correspondence should be addressed: Dr Lutz Jäncke, Ph.D., Institute of General Psychology, Ottovon-Guericke-Universität Magdeburg, Lennéstraße 6, D-39016 Magdeburg, Germany. Tel.: + +49-391-67-14807 or, 14842; Fax: + +49-391-67-14815. blood flow in humans using PET [9, 28] and fMRI [7]. The present study measures fMRI responses in the auditory cortex during verbal and non-verbal auditory stimulation in which the stimulus intensity was varied across conditions in an attempt to answer the question of whether stimulus intensity might influence the fMRI response in the auditory cortex.

\section{Methods}

Fourteen volunteers (13 men and one woman), ranging in age from 20-39 years, with no history of neurological or audiological illness were studied. Hand preference was determined by observing each subject's performance of twelve unimanual and bimanual tasks taken from Annett's handedness questionnaire [1]. All participants used their right hand for all tasks (with "either" preferences being acceptable for unscrewing a jar lid, holding the top of a shovel, dealing cards, or holding the top of a broom). After a full explanation of the nature and risks of the research, subjects gave informed written consent for all studies according to a protocol approved by the Ethics Committee of the Heinrich-Heine University, Düsseldorf.

\section{General methods}

Functional MR images were acquired using a 1.5 Tesla Siemens MRI system (SIEMENS Magnetom Vision, Erlangen, 
Germany), equipped with echo planar imaging (EPI) capability and a standard radiofrequency (RF) head coil for transmit and receive. Pulse sequence parameters were as follows: gradient echo EPI; repetition time $(\mathrm{TR})=5 \mathrm{~s}$; echo time $(\mathrm{TE})=66 \mathrm{~ms}$; field of view $(\mathrm{FOV})=200 \times 200 \mathrm{~mm}$; flip angle (SONDZEICHEN $97\left([\mathrm{gkal})=90^{\circ}\right.$; matrix size $=64 \times 64$; in-plane resolution $=3.125 \times 3.125 \mathrm{~mm}$; slice thickness $=3.0 \mathrm{~mm}$; interslice gap $=0.3 \mathrm{~mm}$. Using a mid-sagittal scout image, 16 axial slices were oriented in the anterior-posterior commissure (ACPC) plane, with the lowermost slice positioned to be $20 \mathrm{~mm}$ below the AC-PC line. In addition, high-resolution, T1-weighted anatomical images of the entire brain were obtained in $3 \mathrm{D}$ using the mp-rage (magnetisation-prepared, rapid acquisition gradient echo) pulse sequence with the following parameters: $\mathrm{TR}=40 \mathrm{~ms} ; \mathrm{TE}=5 \mathrm{~ms} ; \alpha=40^{\circ} ; 1$ excitation; $\mathrm{FOV}=230$ $\mathrm{mm}$; matrix $=256 \times 256 ; 128$ sagittal slices with $1.25 \mathrm{~mm}$ slice thickness.

During scanning the room lights were dimmed and the subjects' eyes were open. Auditory stimuli were presented binaurally using a digital playback system, a magnetically shielded transducer system and air conduction through paired plastic tubes. The air conduction system terminated in tightly occlusive headphones allowing unimpeded conduction of the stimulus with good suppression of ambient scanner noise by about 20 dB.

During each experimental condition, a series of 51 images were acquired. Each series consisted of multiple periods of "baseline" (OFF), during which subjects heard only the ambient machine noise, alternating with periods of "activation" $(\mathrm{ON})$, during which prepared auditory stimuli were delivered. Each series began with three baseline images (15 s interval), followed by 48 images during which "rest" alternated with "activation" every $30 \mathrm{~s}$ (60 s/cycle, 12 images/cycle, 4 cycles). The total duration of each image series was about $4 \mathrm{~min}$.

\section{Auditory stimuli and experimental conditions}

During Experiment 1, nine subjects (8 men and 1 woman) received verbal stimuli. Stimuli were 16-bit, digitally-sampled, consonant-vowel (CV) syllables (/ka/,/ta/,/pa/,/ga/,/da/,/ba/) recorded by a trained phonetician. The onset, duration, intensity, click-free onset and offset, as well as fundamental frequency of the stimuli were edited and synchronized by means of a speech editor. The duration of syllables ranged from 310 $360 \mathrm{~ms}$, depending on voice onset times, with a vowel duration of $300 \mathrm{~ms}$. Voice onset times (in ms) for the stops were approximately $\mathrm{k}=60, \mathrm{t}=50, \mathrm{p}=40, \mathrm{~g}=20, \mathrm{~d}=15, \mathrm{~b}=10$. These syllables were randomized. One-third of the trials consisted of the syllable / $\mathrm{ta} /$ which served as target in the latter experiments. The interval between syllables was $1 \mathrm{~s}$. Subjects were instructed to respond to any occurrence of the target syllable, /ta/, by briefly lifting the index finger of their left hand. Because a plethysmograph was fixed on the index finger, finger movement in the magnetic field of the scanner produced a signal on a monitor outside the magnet enabling registration of subject responses. Three experimental conditions were performed. During each experimental condition the auditory stimuli were presented with different intensities, namely, (i) $75 \mathrm{~dB}$, (ii) $85 \mathrm{~dB}$, or (iii) $95 \mathrm{~dB}$. The order of experimental conditions was randomized across the nine subjects.

During Experiment 2, five further subjects (all male) were presented with non-verbal stimuli during scanning. Stimuli were 16-bit, digitally-sampled tones (pure sine waves: 2000, 1000, $800,600,400$ and $200 \mathrm{~Hz}$ ) with $500 \mathrm{~ms}$ duration each. The order of tones was randomized for each subject. One-third of the trials consisted of the $1000 \mathrm{~Hz}$ tones which served as target tones. The interval between tones was $1 \mathrm{~s}$. Subjects were instructed to respond to any occurrence of the target tone by briefly lifting the index finger of their left hand. We applied the discrimination task in both experiments in order to force the subjects to attend to the stimuli. Experiments previously performed in our laboratory revealed that ignoring or passively listening to auditory stimuli strongly influenced the BOLD response in the auditory cortex [18]. As in Experiment 1, there were also three experimental conditions which were randomized across subjects. During each condition the auditory stimuli were presented with different intensities ((i) $75 \mathrm{~dB}$, (ii) $85 \mathrm{~dB}$, or (iii) $95 \mathrm{~dB})$.

Because of the limitations involved in taking an SPL meter into an MRI scanner, the intensity of the stimuli (mean signal intensity (SPL) during a $30 \mathrm{~s}$ epoch) was determined outside the scanner using an artificial head (Bruel \& Kjaer KA637) wearing the headphones. Frequency analysis of the speech sounds revealed the typical fundamental frequencies for the vowel $/ \mathrm{a} /(800 \mathrm{~Hz}[-33 \mathrm{~dB}]$ and $1200 \mathrm{~Hz}[-34 \mathrm{~dB}])$, the fundamental frequency of the speaker $(134 \mathrm{~Hz}[-37 \mathrm{~dB}])$, and the corresponding overtones.

The noise level within the MRI scanner was measured 1 $\mathrm{m}$ from the bore of the scanner magnet with a capacitance microphone revealing a rms level of 90-100 dB. Because the attenuation factor of the headphones was about $20 \mathrm{~dB}$, the average intensity of the scanner noise perceived by the subjects was estimated to be about $70-80 \mathrm{~dB}$. Frequency analysis of the noise generated by the EPI sequence recorded in the scanner revealed five prominent frequencies. The corresponding amplitudes relative to the maximum amplitude were: $612 \mathrm{~Hz}(-15$ dB), $1847 \mathrm{~Hz}(-29 \mathrm{~dB}), 3036 \mathrm{~Hz}(-21 \mathrm{~dB}), 3365 \mathrm{~Hz}(-37 \mathrm{~dB})$, and $3644 \mathrm{~Hz}(-36 \mathrm{~dB})$. This analysis revealed an overlap of the prominent frequencies of the EPI-noise with prominent frequencies of the stimuli only for the $600 \mathrm{~Hz}$ tone. However, the $600 \mathrm{~Hz}$ tone is not a target tone in the monitoring paradigm. Thus, masking effects should only be minimal. In order to further reduce possible masking effects of scanner noise on the experimental stimuli, a TR of $5 \mathrm{~s}$ was adopted resulting in a non-masked presentation of the stimuli in $60 \%$ of the fMRI measurement time (measurement of one 16-slice EPI scan lasts about $2 \mathrm{~s}$, resulting in $3 \mathrm{~s}$ intervals of silence between scans).

\section{Image analysis}

Image analysis was performed on a SPARC 20 workstation (Sun Microsystems) using MATLAB (Mathworks Inc., Natiek, MA, U.S.A.) and SPM96b software (SPM software, MRC Cyclotron Unit, London, U.K.), [11]. The first three images of each time-series, during which the MR signal reaches a steadystate, were discarded. The 48 remaining volume images of each condition were automatically realigned to the first image to correct for head movement between scans [10]. The images of the three conditions were then coregistered and transformed into a standard stereotaxic space corresponding to the atlas of Talairach and Tournoux [33], using the intercommissural line as the reference plane for the transformation. This normalizing spatial transformation matches each scan (in a least-squares sense) to a template image that already conforms to standard space. The procedure starts with a 12-parameter affine transformation and a six-parameter three-dimensional quadratic (or second order) deformation followed by non-linear (plastic) deformations on a slice by slice basis using Fourier-like basis functions; the parameters are estimated using a least-squares approach after linearizing the problem [10]. In this space one pixel represents $4 \times 4 \mathrm{~mm}$ in the $x$ and $y$ dimensions, with an interplanar distance of $4 \mathrm{~mm}$, allowing direct cross-reference to the atlas topography. These stereotaxically transformed functional data sets from each subject were smoothed slightly with a Gaussian filter of root-mean-square radius of $4 \mathrm{~mm}$ to improve the signal-to-noise ratio and to approximate normal 
distribution of the data. Voxels that had values greater than 0.8 of the volume mean in all the images were selected to restrict analysis to intracranial regions. The effects of global (whole volume) activity and time were removed using linear regression and sine/cosine functions as confounds (up to a maximum of 2.5 cycles per 48 scans). Removing the latter confounds corresponds to high-pass filtering the time series to remove low frequency artifacts due to cardio-respiratory and other cyclical components.

\section{Statistical parameter mapping}

The stereotaxically normalized fMRI time-series data of each subject were analysed separately. Significantly activated pixels were searched for by using the "General Linear Model" approach for time-series data suggested by Friston et al. [1115, 27, 36]. An ANOVA was calculated for each voxel of the functional data set comparing the three intensity blocks with the three rest blocks. The resulting $F$ maps of those voxels with significantly increased signals $(P<0.01)$ were converted to $Z$ maps and an algorithm based on the method derived by Friston et al. [15] was used to determine the size of each activated "cluster", or region of contiguously activated voxels and the probability of each region, based on its spatial extent. Only clusters with a probability of $P<0.05$ (corrected for multiple comparisons) and a height threshold of $Z=3.09$ (not corrected for multiple comparisons) were considered for further evaluation. The activated voxels surviving this procedure were superimposed on "SPM brain projections" and on individual stereotaxically normalized high-resolution MR-anatomical scans. Because the clusters of activated voxels covered a wide range of the auditory cortex, we additionally defined regions of interest (ROI) in each hemisphere to account for the diversity of the auditory cortex (primary, immediate and secondary auditory cortex). These ROIs were defined according to the Talairach atlas and prominent sulcal and gyral landmarks (Heschl's gyrus, sylvian fissure, ramus posterior ascendens, ramus posterior descendens, and superior temporal sulcus [19, 31]: (1) the primary and immediate auditory area (Broadmann area, BA 41/42) and (2) the auditory association area (BA 22). However, the exact spatial delineation of the auditory cortices is currently not possible for the following reasons: (i) the spatial extent of the cytoarchitectonic area of the primary and immediate auditory cortex is currently unknown, (ii) the exact delineation of the anatomical landmarks was not possible because of the limited spatial resolution of the normalized fMRI images (4 $\mathrm{mm}^{3}$ ), (iii) the angulated shape of the Heschl's gyri and the sylvian fissure varied considerably in horizontal and vertical directions within subjects (left vs right hemisphere) and between subjects. Thus, we defined as primary and immediate auditory cortex a region covering the Heschl's gyrus (as defined by the Heschl's sulcus and the first transverse sulcus) on at least two horizontal planes taken from the Talairach atlas $(Z=12$ and $Z=8$ ). The secondary auditory cortex was defined as the area posterior to the primary and immediate auditory cortex extending into the inferior and superior directions from plane $Z=0$ and to $Z=20$ more posteriorily. Thus, our ROI definitions and Broadman area (BA) designations should be taken only as approximate. For these ROIs, numbers of significantly activated voxels, as well as mean signal intensity changes relative to baseline were determined for each subject individually (individual analysis).

In order to present pictorially the overall pattern of activation across subjects the stereotaxically transformed functional data sets were averaged across subjects (group analysis). As for the individual analyses only pixels passing a height threshold of $Z=3.09$ and a cluster threshold of $P<0.05$ were considered as significant. The activated voxels surviving this procedure were superimposed on "SPM brain projections" (Fig.1a and 1b). From these analyses stereotaxically coordinates of centerof-mass of activated regions were determined and are presented in Tables 1 and 2.

\section{Results}

Subjects were able to perform the discrimination tasks quite well (average hit rates for verbal stimuli: 75 $\mathrm{dB}=84 \%, 85 \mathrm{~dB}=84 \%, 95 \mathrm{~dB}=87 \%$; for non-verbal

\section{verbal}

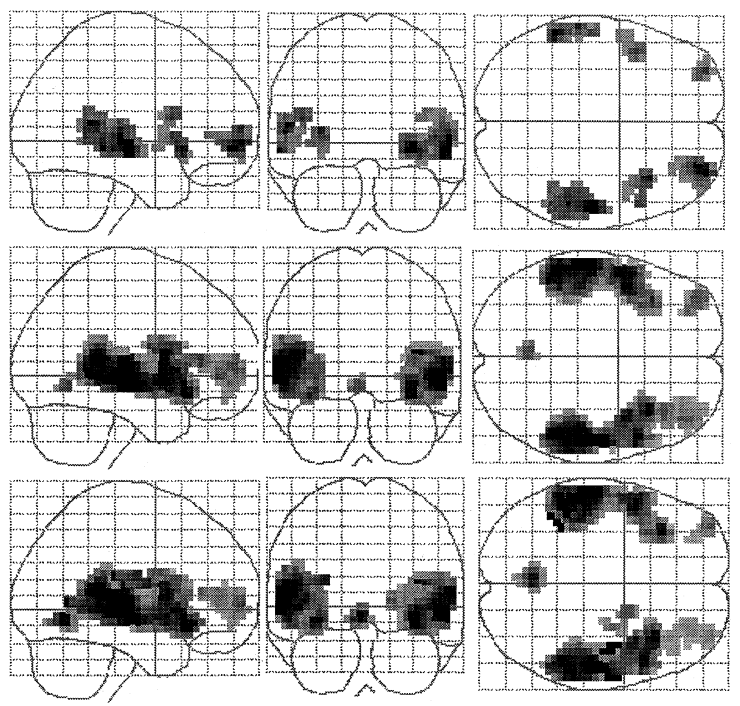

$75 \mathrm{~dB}$

\section{non-verbal}



Fig. 1. Schematic representation of mean activated areas on stereotaxically normalized SPM glass brains: (a) verbal stimuli study, (b) non-verbal stimuli study. The three brain projections viewing the brain from above (transverse), the right (sagittal) and the back 
Table 1. Significant activation foci for the three stimulus intensity conditions (verbal stimuli) obtained in the group analysis. We report only positive activation peaks located within the parenchyma. Stereotaxic center-of-mass coordinates refer to medial-lateral position $(x)$ relative to midline (positive $=$ right), anterior-posterior position $(y)$ relative to the anterior commissure (positive $=$ anterior), and superior-inferior position $(z)$ relative to the commissural line (positive $=$ superior)

\begin{tabular}{|c|c|c|c|c|c|c|}
\hline \multirow{2}{*}{$\begin{array}{l}\text { Stimulus } \\
\text { intensity }\end{array}$} & \multicolumn{4}{|c|}{ Coordinates (mm) } & \multirow[b]{2}{*}{ Atlas structure at center-of-mass } & \multirow{2}{*}{$\begin{array}{c}\text { Broadmar } \\
\text { area }\end{array}$} \\
\hline & $x$ & $y$ & $z$ & $Z$-score & & \\
\hline \multirow[t]{9}{*}{$75 \mathrm{~dB}$} & 56 & -20 & 4 & 8,6 & Right transverse temporal gyrus (Heschl) & $41 / 42$ \\
\hline & -56 & -44 & 12 & 6,4 & Left superior temporal gyrus & 22 \\
\hline & 56 & -16 & 4 & 8,1 & Right superior temporal gyrus & 22 \\
\hline & -56 & -22 & 12 & 3,6 & Left transverse temporal gyrus (Heschl) & $41 / 42$ \\
\hline & 28 & 56 & -4 & 6,5 & Right middle frontal gyrus & 10 \\
\hline & 36 & 20 & -4 & 5,9 & Right inferior frontal gyrus & 47 \\
\hline & -44 & 12 & 16 & 5,4 & Left inferior frontal gyrus & 47 \\
\hline & 24 & 40 & 4 & 4,1 & Right inferior frontal gyrus & 45 \\
\hline & -56 & 12 & -4 & 3,5 & Left inferior frontal gyrus & 47 \\
\hline \multirow[t]{9}{*}{$85 \mathrm{~dB}$} & -56 & -20 & 4 & 8,6 & Left superior temporal gyrus & 22 \\
\hline & -56 & -20 & 12 & 7,9 & Left transverse temporal gyrus (Heschl) & $41 / 42$ \\
\hline & 56 & -36 & 4 & 7,8 & Right superior temporal gyrus & 22 \\
\hline & 56 & -24 & 12 & 7,7 & Right transverse temporal gyrus (Heschl) & 41 \\
\hline & 36 & 20 & -8 & 7,7 & Right inferior frontal gyrus & 47 \\
\hline & 40 & 41 & 6 & 7,5 & Right precental gyrus & 6 \\
\hline & 36 & 32 & 12 & 6,1 & Right inferior frontal gyrus & 10 \\
\hline & -36 & 56 & 4 & 5,9 & Left middle frontal gyrus & 10 \\
\hline & -4 & -60 & -8 & 5,5 & Cerebellum & \\
\hline \multirow[t]{9}{*}{$95 \mathrm{~dB}$} & 56 & -20 & -4 & 8,7 & Right middle temporal gyrus & 21 \\
\hline & -56 & -20 & 4 & 8,5 & Left superior temporal gyrus & 22 \\
\hline & 60 & -36 & 8 & 7,8 & Right superior temporal gyrus & 22 \\
\hline & 56 & -24 & 12 & 7,7 & Right transverse temporal gyrus (Heschl) & $41 / 42$ \\
\hline & -56 & -20 & 12 & 7,6 & Left transverse temporal gyrus (Heschl) & $41 / 42$ \\
\hline & 36 & 20 & -8 & 7,9 & Right inferior frontal gyrus & 47 \\
\hline & -4 & -60 & -4 & 7,4 & Cerebellum & \\
\hline & -36 & 60 & 4 & 4,9 & Left middle frontal gyrus & 10 \\
\hline & 36 & 32 & 12 & 4,6 & Right inferior frontal gyrus & 46 \\
\hline
\end{tabular}

Table 2. Significant activation foci for the three stimulus intensity conditions (non-verbal stimuli) obtained in the group analysis. We report only positive activation peaks located within the parenchyma. Stereotaxic center-of-mass coordinates refer to mediallateral position $(x)$ relative to midline (positive $=$ right), anterior-posterior position $(y)$ relative to the anterior commissure $($ positive $=$ anterior $)$ and superior-inferior position $(z)$ relative to the commissural line (positive $=$ superior)

\begin{tabular}{|c|c|c|c|c|c|c|}
\hline \multirow{2}{*}{$\begin{array}{l}\text { Stimulus } \\
\text { intensity }\end{array}$} & \multicolumn{4}{|c|}{ Coordinates (mm) } & \multirow[b]{2}{*}{ Atlas structure at center-of-mass } & \multirow{2}{*}{$\begin{array}{c}\text { Broadman } \\
\text { area }\end{array}$} \\
\hline & $x$ & $y$ & $z$ & $Z$-score & & \\
\hline \multirow[t]{4}{*}{$75 \mathrm{~dB}$} & -52 & -28 & 16 & 7,0 & Left superior temporal gyrus & 22 \\
\hline & -56 & -24 & 12 & 4,6 & Left transverse temporal gyrus (Heschl) & $41 / 42$ \\
\hline & 64 & -12 & 4 & 7,3 & Right superior temporal gyrus & 22 \\
\hline & 56 & 28 & 16 & 6,6 & Right inferior frontal gyrus & 46 \\
\hline \multirow[t]{6}{*}{$85 \mathrm{~dB}$} & -44 & -20 & 4 & 6,7 & Left superior temporal gyrus & 22 \\
\hline & 64 & -24 & 8 & 7,7 & Right superior temporal gyrus & 22 \\
\hline & 60 & -20 & 12 & 4,3 & Right transverse temporal gyrus (Heschl) & $41 / 42$ \\
\hline & -56 & -20 & 12 & 3,6 & Left transverse temporal gyrus (Heschl) & $41 / 42$ \\
\hline & -40 & 24 & 0 & 4,9 & Left inferior frontal gyrus & 45 \\
\hline & 156 & 32 & 16 & 7,0 & Right inferior frontal gyrus & 45 \\
\hline \multirow[t]{6}{*}{$95 \mathrm{~dB}$} & -44 & -20 & 6 & 6,8 & Left superior temporal gyrus & 22 \\
\hline & 56 & -24 & 8 & 7,6 & Right superior temporal gyrus & 22 \\
\hline & 52 & -16 & 12 & 4,1 & Right transverse temporal gyrus (Heschl) & $41 / 42$ \\
\hline & -56 & -20 & 12 & 3,5 & Left transverse temporal gyrus (Heschl) & $41 / 42$ \\
\hline & -40 & 24 & 2 & 5,1 & Left inferior frontal gyrus & 45 \\
\hline & 56 & 32 & 12 & 6,5 & Right inferior frontal gyrus & 45 \\
\hline
\end{tabular}


Table 3. Mean number of activated voxels (and standard deviations) for BA22 for each condition (75, 85 and $95 \mathrm{~dB}$ ), both hemispheres (1Hem, rHem: left and right hemisphere) and for each study (verbal and non-verbal)

\begin{tabular}{|c|c|c|c|c|c|c|}
\hline Study & $\begin{array}{l}75 \mathrm{~dB} \\
1 \mathrm{Hem}\end{array}$ & $\begin{array}{l}85 \mathrm{~dB} \\
1 \mathrm{Hem}\end{array}$ & $\begin{array}{l}95 \mathrm{~dB} \\
1 \mathrm{Hem}\end{array}$ & $\begin{array}{l}75 \mathrm{~dB} \\
\text { rhem }\end{array}$ & $\begin{array}{l}85 \mathrm{~dB} \\
\text { rhem }\end{array}$ & $\begin{array}{l}95 \mathrm{~dB} \\
\text { rhem }\end{array}$ \\
\hline \multicolumn{7}{|l|}{ Verbal } \\
\hline Mean & 50.3 & 202.2 & 422.2 & 74.6 & 237.3 & 429.6 \\
\hline SD & 31.9 & 92.1 & 331.9 & 45.2 & 59.9 & 347.4 \\
\hline \multicolumn{7}{|c|}{ Non-verbal } \\
\hline Mean & 47.6 & 39.6 & 133.2 & 78.0 & 103.8 & 180.0 \\
\hline SD & 80.6 & 56.1 & 71.1 & 114.9 & 13.0 & 88.0 \\
\hline \multicolumn{7}{|l|}{ Total } \\
\hline Mean & 49.4 & 144.1 & 319.0 & 75.8 & 189.6 & 340.4 \\
\hline SD & 51.2 & 112.81 & 300.0 & 73.0 & 99.4 & 303.4 \\
\hline
\end{tabular}

stimuli: $75 \mathrm{~dB}=76 \%, 85 \mathrm{~dB}=75 \%, 95 \mathrm{~dB}=69 \%$ ). The target syllables were discriminated significantly better than the target tones $(F(1,12)=19,8, P<0.001)$. Stimulus intensity only had an effect on the discrimination of verbal stimuli but not on non-verbal stimuli $(F(2,24)=10.5, P<0.001)$.

\section{Group analysis}

Contrasting fMRI signals obtained under baseline conditions (OFF period) with those obtained during activation conditions ( $\mathrm{ON}$ periods) revealed significantly activated voxels mainly in the superior temporal gyrus (STG) of both hemispheres (Fig. 1). The center-of-mass of the activation in these regions fell well within the secondary and primary auditory cortices (Tables 1 and 2) of both hemispheres. Beside these activations found in the auditory cortex there were additionally activated regions mainly in the inferior and middle frontal gyrus (BA 47, 46, 45, 4) the left precentral gyrus (BA 6), the left middle temporal gyrus (BA 21), the anterior part of the left superior temporal gyrus (BA 22/38), and the cerebellum.

\section{Individual analysis}

Because the main interest of the present work was to study the BOLD response in the auditory cortex, we shall concentrate for the individual analysis on activation in these areas. The number of activated voxels in both auditory ROIs (BA 41/42 and BA 22) were subjected to separate $3 \times 2 \times 2$ ANOVAs (intensity: 75,85 and $95 \mathrm{~dB}$, hemisphere: left and right and study: verbal vs nonverbal) with repeated measurements on two factors (intensity and hemisphere). The mean number of voxels for both ROIs are presented in Tables 2 and 3. Because there were inhomogeneities of variances we also calculated the statistics $\mathrm{ETA}^{2}$ in order to demonstrate the more descriptive approach of the present analysis and to describe the variance which accounted for a specific effect. The threelevel intensity factors were decomposed into two orthogonal contrasts testing for differences between $75 \mathrm{~dB}$ (low

Table 4. Mean number of activated voxels (and standard deviations) for BA41/42 for each condition (75, 85 and $95 \mathrm{~dB}$ ), both hemispheres (lHem, rHem: left and right hemisphere) and for each study (verbal and nonverbal)

\begin{tabular}{lcccccc}
\hline Study & $\begin{array}{c}75 \mathrm{~dB} \\
1 \mathrm{Hem}\end{array}$ & $\begin{array}{l}85 \mathrm{~dB} \\
\text { 1Hem }\end{array}$ & $\begin{array}{c}95 \mathrm{~dB} \\
1 \mathrm{Hem}\end{array}$ & $\begin{array}{c}75 \mathrm{~dB} \\
\text { rhem }\end{array}$ & $\begin{array}{c}85 \mathrm{~dB} \\
\text { rhem }\end{array}$ & $\begin{array}{c}95 \mathrm{~dB} \\
\text { rhem }\end{array}$ \\
\hline $\begin{array}{l}\text { Verbal } \\
\text { Mean }\end{array}$ & 21.0 & 74.7 & 100.0 & 16.1 & 47.9 & 67.4 \\
$\quad \begin{array}{l}\text { Std } \\
\text { Non-verbal }\end{array}$ & 31.5 & 48.3 & 53.9 & 25.5 & 41.5 & 45.8 \\
$\quad \begin{array}{l}\text { Mean } \\
\text { Std }\end{array}$ & 39.6 & 74.4 & 99.0 & 46.4 & 54.8 & 76.6 \\
$\begin{array}{l}\text { Total } \\
\text { Mean }\end{array}$ & 72.8 & 45.9 & 38.3 & 52.5 & 45.1 & 22.0 \\
$\quad$ Std & 27.6 & 74.6 & 99.6 & 26.9 & 50.4 & 70.7 \\
\hline
\end{tabular}


intensity) and the average of $85 \mathrm{~dB}$ and $95 \mathrm{~dB}$ (high intensity), as well as differences between $85 \mathrm{~dB}$ and $95 \mathrm{~dB}$. For the number of voxels counted in BA 22 we found a significant intensity effect with more voxels activated under higher intensities $((95 \mathrm{~dB}+85 \mathrm{~dB}) / 2$ : $\left.F(1,12)=11.3, P<0.001, \mathrm{ETA}^{2}=0.48\right)$. There was also an interaction between intensity and study for the difference between low and high intensities $(F(1,12)=5.1$, $\left.P=0.04, \mathrm{ETA}^{2}=0.29\right)$ reflecting the sharper rise in the number of voxels from low to high intensity for verbal stimuli. In addition, we found that more voxels were activated in the right hemisphere $(F(1,12)=10.9$, $\left.P<0.001, \mathrm{ETA}^{2}=0.47\right)$ and during the stimulation with verbal stimuli $\left(F(1,12)=4.8, P<0.05, \mathrm{ETA}^{2}=0.29\right)$. The number of voxels activated by the 85 and $95 \mathrm{~dB}$ stimuli differed slightly $(F(1,12)=4.1, \quad P<0.06$, $\left.\mathrm{ETA}^{2}=0.26\right)$ with more voxels activated under $95 \mathrm{~dB}$ stimulation. For this contrast there were also significant hemisphere $\left(F(1,12)=7.5, P<0.02, \mathrm{ETA}^{2}=0.39\right)$ and study effects $\left(\left(F(1,12)=5.6, P=0.03\right.\right.$, ETA $\left.^{2}=0.32\right)$ reflecting more voxels activated in the right hemisphere and during verbal stimulation.

In BA 41/42 we also found more voxels activated by high intensity stimuli than by low intensity stimuli $\left.F(1,12)=21.9, \quad P<0.001, \quad \mathrm{ETA}^{2}=0.65\right)$. Even the difference between number of voxels activated under the 95 and $85 \mathrm{~dB}$ conditions differed significantly $\left(F(1,12)=4.5, P<0.05, \mathrm{ETA}^{2}=0.27\right)$.

Finally, there was also a hemisphere difference yielding more voxels activated in the left hemisphere $\left(F(1,12)=6.5, P<0.02, \mathrm{ETA}^{2}=0.35\right)$. In contrast to the aforementioned region (BA22), we found no difference for the number of activated voxels during verbal and non-verbal stimulation. Figure 2 shows the mean number of active voxels for both ROIs, averaged over all subjects, separately, for both experiments. It is obvious that the spatial extent of activated volume in the auditory cortex strongly varied with the intensity of auditory stimuli (Figs 1 and 2). Mean signal intensity for the ROIs varied between $0.6-1.8 \%$ (relative to baseline), but there was no significant effect of stimulus intensity, hemisphere, or study on the signal intensity.

\section{Discussion}

During both auditory tasks we observed activation in the primary and secondary auditory cortex (BA 41, 42, and 22), consistent with the findings of previous fMRI studies $[4,7,8,23,32,34]$ and earlier work with PET [17, $22,24,26,28,35,38]$. The present study again demonstrates that verbal and non-verbal auditory stimulation evokes a BOLD response in the primary and secondary auditory cortex despite a significant background of acoustic noise from the gradient system of the scanner.

However, the main finding of the present study is a robust and highly significant stimulus intensity effect on the spatial extent of activated volume in auditory cortex. The spatial extent of the activated volume increases with increasing level of stimulus intensity. The results of this study are in contrast to those of an earlier fMRI study [23] which found no influence of stimulus intensity on the BOLD response in auditory cortex. It is difficult to make a direct comparison of our work with the above-mentioned report. However, a number of differences may help to explain why we obtained an intensity-dependent activation effect which was absent in the earlier work. Millen et al. did not actively control for head movement artifacts although head movements are, unfortunately, all too common in fMRI experiments despite the use of head restraints and with subjects who are fully aware of the effects of head movements on the data. Artifacts resulting from head movement are a serious confound and can lead to false identification of activated regions
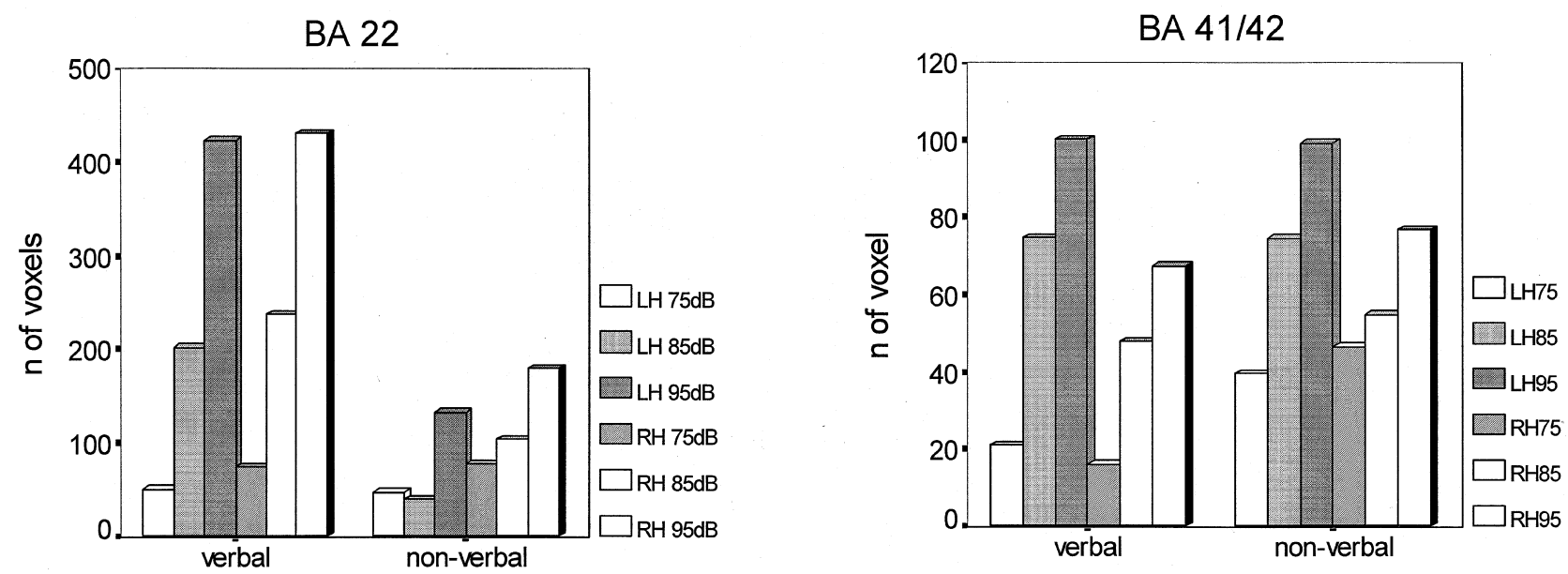

Fig. 2. (a) Mean number of activated voxels in BA 22 and (b) in BA 41/42 under three stimulus intensity conditions and for both studies (verbal and non-verbal stimuli). 
and general misinterpretation of results [16]. Millen et al. used a rather short TR of $2 \mathrm{~s}$ which leads to saturation effects and necessitates the use of correction for spin history effects; no such correction was performed in the previous work and was rendered unnecessary here by the use of a long TR period ( $5 \mathrm{~s}$ ) which also decreases the masking influence of acoustic scanner noise [30] since there are noise-free periods in the paradigm during which stimuli are presented. Possible differences may also arise from the use of a $15 \mathrm{~mm}$ slice thickness by Millen et al. as compared with $3 \mathrm{~mm}$ thick slices in this study. Additionally, it has been shown elegantly by Bandettini et al. [2] that the acoustic noise generated by the gradient system does result in activation of the auditory cortex. The activation signal in the above-mentioned study falls to zero in about $5 \mathrm{~s}$, further demonstrating the need to avoid short TR times for fMRI studies of the auditory system. There is also another substantive procedural difference which makes direct comparisons difficult. In our study, the auditory stimuli ranged between 75-95 dB while they were in the range of $20-50 \mathrm{~dB}$ in the Millen et al. study. It is indeed remarkable that they were able to evoke reliable BOLD responses in the auditory cortex by using such low intensity stimuli. Our own experience is that reducing the stimulus intensity to $60 \mathrm{~dB}$ reduces the signal in auditory cortex dramatically, mostly making it impossible to detect statistically significant activations. Hence, as our lowest intensity, we used $75 \mathrm{~dB}$ in order to reliably evoke BOLD responses in all subjects. It should be noted that recent studies of auditory stimuli in fMRI experiments used high-intensity stimuli, often greater than $90 \mathrm{~dB}[5,6,8]$ in order to reduce the masking effect of the scanner noise. Millen at al. inconsistently varied the different intensities across the experimental conditions, making it difficult to exclude possible carry over effects.

It is known that fMRI signals are influenced by changed oxygenation levels of brain tissue as well as to oxygenation in draining veins. However, because we used a relatively long TR time $(5 \mathrm{~s})$ in order to reduce saturation effects and signal intensity changes associated with inflow effects in draining veins, it is very likely that the fMRI signals measured here at least indirectly reflects neuronal activity. Thus, the fMRI signals monitored here probably indicate intensity and/or loudness coding in the auditory processing system. From basic psychophysical experiments it is known that up to a stimulus intensity of about $60 \mathrm{~dB}$ above threshold, intensities are neurally signaled as changes in firing rates of neurons with similar center frequencies (CF). For intensities greater than $60 \mathrm{~dB}$ above threshold most neurons are saturated (firing at their maximum rate). Only a small fraction of these neurons have thresholds much higher than the majority. These "high threshold" neurons are additionally activated under high intensity stimulation coding intensity with increasing neural firing rate $[20,21]$. Because the proportion of nerve fibers with wide dynamic range ("high threshold" fibers) is only about $10 \%$ and changes in intensity result in only small changes in firing rates a further mechanism for the coding of intensity changes at high intensities is necessary. One very likely mechanism is that the neural excitation pattern of the stimulus spreads with increasing intensity. At high intensities the majority of neurons at the center of the pattern would be saturated, but changes in intensity could still be signaled by changes in the firing rates of neurons at the edges of the pattern. Taken together, increasing firing rate of "high threshold" neurons and spreading of activations to neighboring neurons might be associated with an increased spatial extent and fMRI intensity of neural activity. However, the intensity effect was smaller for the high intensity stimuli ( $95 \mathrm{~dB}>85 \mathrm{~dB})$ than for the difference between low $(75 \mathrm{~dB})$ and high (average of 85 and $95 \mathrm{~dB})$ intensity stimuli. This result might indicate a possible saturation of cerebral blood flow and/or of an increase in spatial extent of neural activity. A final comment should be made on the relation between the psychophysical measures of target detection and the BOLD response under the three intensity conditions. There was a drop in target detection performance under the low intensity condition only for the verbal but not for the non-verbal stimuli. Thus, the intensity dependent BOLD response does not solely depend on the discriminability of stimuli. However, from psychophysical studies it is known that the discriminability of auditory stimuli increases with increasing stimulus intensity implying a possible interaction between stimulus intensity and stimulus discriminability.

A further interesting result of the present study is that verbal stimuli evoked a greater extent of activity within the secondary auditory cortex than the non-verbal stimuli. This might indicate that processing of verbal stimuli is performed in more widespread neural networks than the processing of simple tones. This assumption coincides with recent electrophysiological studies on non-human primates demonstrating that complex sounds evoked a more extended activity over the auditory cortex [29]. Interestingly, there was no general difference in terms of activated volume in the primary auditory cortex, suggesting that similar networks are involved in the primary processing of auditory stimuli.

In addition, we found activation in the left and right inferior frontal gyri during the monitoring of verbal and non-verbal stimuli. This result conforms with the suggestions that the superior temporal gyrus and the inferior frontal gyrus form a network specifically associated with the retrieval and rehearsal of auditory information, particulary in the absence of external stimulation [37]. Activation of this auditory network during our tasks parallels findings from a study in which silent rehearsal of letter strings produces bilateral activation of the superior temporal gyrus and the inferior frontal gyrus [25]. This suggested to the authors of the above-referenced study that the articulatory loop of working memory includes a subvocal rehearsal system. This interpretation suggests the possibility that in our study, an internal, auditory representation of the target was sustained in order to be used for comparison with the actual stimuli. It might also be 
possible that the comparison of the actual syllable with the target syllable is only possibly via this articulatory loop since both stimuli types are only present auditorily.

\section{Conclusion}

The present fMRI study demonstrates that the spatial extent of activation in superior temporal gyrus in an auditory discrimination task strongly depends on stimulus intensity. The spatial extent of activated volume increases with increasing stimulus intensity. The results suggest that increasing stimulus intensity is associated with increasing cerebral blood flow, most likely indicating spreading excitation of adjacent neurons at the edge of best-firing neurons. The present results may help in the understanding of cortical coding of increasing stimulus intensity and may help to design future fMRI experiments applying auditory stimuli.

Acknowledgements - This work was supported by the grants from the Deutsche Forschungsgemeinschaft (JA 737/4-1 and JA 737/5-1) awarded to L.J.

\section{References}

1. Annett, M., A classification of hand preference by association analysis. Br. J. Psychol., 1970, 61, 303321

2. Bandettini, P. A., Jesmanowicz, A., Van Kylen, J., Birn, R. M. and Hyde, J. S., FMRI of scanner noise induced auditory cortex activation. Proceedings of the International Society of Magnetic Resonance in Medicine, 1997, 349 (Abstract).

3. Binder, J. R., Frost, J. A., Hammeke, T. A., Cox, R. W., Rao, S. M. and Prieto, T., Human brain language areas identified by functional magnetic resonance imaging. J. Neurosci., 1997, 17, 353-362.

4. Binder, J. R., Frost, J. A., Hammeke, T. A., Rao, S. M. and Cox, R. W., Function of the left planum temporale in auditory and linguistic processing. Brain, 1996, 119, 1239-1247.

5. Binder, J. R., Rao, S. M., Hammeke, T. A., Frost, J. A., Bandettini, P. A. and Hyde, J. S., Effects of stimulus rate on signal response during functional magnetic resonance imaging of auditory cortex. Brain Res. Cogn. Brain Res., 1994, 2, 31-38.

6. Binder, J. R., Rao, S. M., Hammeke, T. A., Frost, J. A., Bandettini, P. A., Jesmanowicz, A. and Hyde, J. S., Lateralized human brain language systems demonstrated by task subtraction functional magnetic resonance imaging. Arch. Neurol., 1995, 52, 593-601.

7. Binder, J. R., Rao, S. M., Hammeke, T. A., Yetkin, F. Z., Jesmanowicz, A., Bandettini, P. A., Wong, E. C., Estkowski, L. D., Goldstein, M. D., Haughton, V. M. et al., Functional magnetic resonance imaging of human auditory cortex [see comments]. Ann. Neurol., 1994, 35, 662-672.

8. Binder, J. R., Swanson, S. J., Hamecke, T. A.,
Mprris, G. L., Mueller, W. M., Fischer, M., Benbadis, S., Frost, J. A., Rao, S. M. and Haughton, V. M., Determination of language dominance using functional MRI: A comparison with the Wada test. Neurology, 1996, 46, 978-984.

9. Fox, P. T. and Raichle, M. E., Stimulus rate determines regional brain blood flow in striate cortex. Ann. Neurol., 1985, 17, 303-305.

10. Friston, K. J., Ashburner, J., Poline, J. B., Frith, C. D., Heather, J. D. and Frackowiack, R. S. J., Spatial realigment and normalzation of images. Hum. Brain Mapp., 1997, 00, 000-000.

11. Friston, K. J., Holmes, A. P., Ashburner, J., and Poline, J. B., World Wide Web http://www.fil.ion. ucl.ac.uk/spm. 1996.

12. Friston, K. J., Holmes, A. P., Poline, J. B., Grasby, P. J., Williman, S. C. R., Frackowiack, R. S. J. and Turner, R., Analysis of fMRI time-series revisited. Neuroimage, 1995, 2, 45-53.

13. Friston, K. J., Holmes, A. P., Worsley, K. P., Poline, J.-B., Frith, C. D. and Frackowiack, R. S. J., Statistical parametric maps in functional imaging: a general linear approach. Hum. Brain Mapp., 1995, 2, 189-210.

14. Friston, K. J., Jezzard, P. and Turner, R., Analysis of Functional MRI Time-Series. Hum. Brain Mapp., 1994, 1, 153-171.

15. Friston, K. J., Worsley, K. J., Frackowiak, R. S. J., Mazziotta, J. C. and Evans, A. C., Assessing the Significance of Focal Activations Using Their Spatial Extent. Hum. Brain Mapp., 1994, 1, 210-220.

16. Hajnal, J. V., Bydder, G. M. and Young, I. R., Stimulus-correlated signals in functional MR of the brain [comment]. AJNR. Am. J. Neuroradiol., 1996, 17, 1011-1012.

17. Herzog, H., Cortical activation by auditory stimulation studied with positron emission tomography. In Functional Neuroimaging, ed. R. W. Thatcher, M. Hallet, T. Zeffiro and E. R. John. Academic Press, New York, pp. 59-67.

18. Jäncke, L., Posse, S., Shah, N. J., Nösselt, T., Schmitz, N. and Müller-Gärtner, H.-W., Attentional factors modify the BOLD-response in the human auditory cortex to auditory stimuli. Neuroimage, 1997, 5, S191 (Abstract).

19. Jäncke, L., Schlaug, G., Huang, Y. and Steinmetz, H., Asymmetry of the planum parietale. NeuroReport, 1994, 5, 1161-1163.

20. Kiang, N. Y. S., A survey of recent developments in the study of auditory physiology. Ann. Otol. Rhinol. Laryngol., 1968, 77, 656-675.

21. Liberman, M. C., Auditory-nerve response from cats raised in a low-noise chamber. J. Acoust. Soc. Am., 1978, 63, 442-455.

22. Mazoyer, B. M., Tzuori, N., Frak, V., Syrota, A., Murayama, N., Levrier, O., Salamon, G., Dehaene, S., Cohen, L. and Mehler, J., The cortical representation of speech. J. Cog. Neurosci., 1993, 5, 467479.

23. Millen, S. J., Haughton, V. M. and Yetkin, Z., Functional magnetic resonance imaging of the central auditory pathway following speech and pure-tone stimuli. Laryngoscope, 1995, 105, 1305-1310. 
24. Naito, Y., Okazawa, H., Honjo, I., Hirano, S., Takahashi, H., Shiomi, Y., Hoji, W., Kawano, M., Ishizu, K. and Yonekura, Y., Cortical activation with sound stimulation in cochlear implant users demonstrated by positron emission tomography. Brain Res. Cogn. Brain Res., 1995, 2, 207-214.

25. Paulesu, E., Frith, C. D. and Frackowiak, R. S., The neural correlates of the verbal component of working memory [see comments]. Nature, 1993, 362, 342-345.

26. Petersen, S. E., Fox, P. T., Posner, M. I., Mintun, M. and Raichle, M. E., Positron emission tomographic studies of the cortical anatomy of single-word processing. Nature, 1988, 331, 585-589.

27. Poline, J. B., Worsley, K. J., Holmes, A. P., Frackowiak, R. S. and Friston, K. J., Estimating smoothness in statistical parametric maps: variability of $p$ values. J. Comput. Assist. Tomogr., 1995. 19, 788-796.

28. Price, C., Wise, R., Ramsay, S., Friston, K., Howard, D., Patterson, K. and Frackowiak, R., Regional response differences within the human auditory cortex when listening to words. Neurosci. Lett., 1992, 146, 179-182.

29. Rauschecker, J. P., Tian, B. and Hauser, M., Processing of complex sounds in the macaque nonprimary auditory cortex. Science, 1995, 268, 111114.

30. Shah, N. J., Jäncke, L., Grosse-Ruyken, M., Posse, S. and Müller-Gärtner, H.-W. How does acoustic masking noise affect fMRI of the auditory cortex during phonetic discrimination? Neuroimage, 1997, 5, S195 (Abstract).

31. Steinmetz, H., Rademacher, J., Jäncke, L., Huang,
Y. X., Thron, A. and Zilles, K., Total surface of temporoparietal intrasylvian cortex: diverging leftright asymmetries. Brain Lang., 1990, 39, 357-372.

32. Strainer, J. C., Ulmer, J. L., Yetkin, F. Z., Haughton, V. M., Daniels, D. L. and Millen, S. J., Functional MR of the primary auditory cortex: an analysis of pure tone activation and tone discrimination [see comments]. AJNR. Am. J. Neuroradiol., 1997, 18, 601-610.

33. Talairach, J. and Tournoux, P. Co-Planar stereotaxic atlas of the human brain. 3-Dimensional proportional system: an approach to cerebral imaging. Thieme Medical Publishers, Inc., New York, 1988.

34. Wessinger, C. M., Buonocore, M. H., Kussmaul, C. L. and Mangun, G. R., Tonotopy in human auditory cortex examined with functional magnetic resonance imaging. Hum. Brain Mapp., 1997, 5, 18-25.

35. Wise, R., Chollet, F., Hadar, U., Friston, K. Hoffner, E. and Frackowiak, R., Distribution of cortical neural networks involved in word comprehension and word retrieval. Brain, 1991, 114, 1803-1817.

36. Worsley, K. J. and Friston, K. J., Analysis of fMRI time-series revisited-again. Neuroimage, 1995, 3, 173-181.

37. Zatorre, R. J., Halpern, A. R., Perry, D. W., Meyer, E. and Evans, A. C., Hearing in the mind's ear: a PET investigation of musical imagery and perception. $J$. Cog. Neurosci., 1996, 8, 29-46.

38. Zatorre, R. J., Jones Gotman, M., Evans, A. C. and Meyer, E., Functional localization and lateralization of human olfactory cortex. Nature, 1992, 360, 339 340 . 